\title{
Gamma Knife Radiosurgery: An Overview of Physics, Chemistry, Biology and Neuro-medicine
}

\author{
KMN UDDIN ${ }^{\mathrm{a}}$, JN ISLAM ${ }^{\mathrm{b}}$
}

\begin{abstract}
Summary:
The gamma knife is a highly specialized treatment unit that provides an advanced sophisticated stereotactic approach to treatment of tumour and vascular malformations within the internal structure of the head. The gamma knife delivers a single high dose of radiation emanating from 201 cobalt60 unit sources. All 201 beam simultaneously intersect at the same time in a pre-defined location. The treatment planning system for gamma knife radiosurgery has been developed using nonlinear programming techniques. The system optimizes the shot sizes, location and weights for gamma knife treatments. Open stereotactic technique in the 1990's was essential for the treatment of a number of functional conditions and cystic space occupying lesions. It has an important part to play in the investigation of tumours and can help to increase the number which are accessible to treatment. It can be employed to guide not only solid instruments but also ionizing irradiation to "masslesion - targets". It is just this combination of stereotactic
\end{abstract}

\section{Introduction}

The gamma knife is a very specialized treatment unit that provides an advanced and sophisticated approach to the treatment of tumour vascular malformations, and pain disorder within the head. Multiple beams of radiation are focused into an approximately spherical volume, from inside a shielded treatment unit, generating high dose shot of radiation. The treatment planning process determines where to centre the shot, how long to expose them for and what size focusing helmets should be used, so as to cover the target with sufficient dosage without damaging normal tissues or surrounding sensitive structures. There

a. Lecturer, Department of Accounting, Jagannath University, Dhaka. And Ph.D. Fellow (Research Centre for Mathematical and Physical Sciences, Chittagong University, Chittagong).

b. Professor Emeritus, Research Centre For Mathematical And Physical Sciences Chittagong University, Chittagong.

Address of Correspondence: Kazi Md. Nasir Uddin, Lecturer, Department of Accounting, Jagannath University, Dhaka. And Ph.D. Fellow (Research Centre for Mathematical and Physical Sciences, Chittagong University, Chittagong),E-mail. : Kazinasiruddin@yahoo.com Received: 8 July, 2009

Accepted: 21 January, 2010 guidance and narrow beam, high energy radiation to precisely defined target, is the basis of gamma knife radiosurgery. The topic on radiological physics presents a broad field, which includes physics of radiation therapy, diagnosis and nuclear medicine. The emphasis is on the basic physical principles which form a common foundation for these areas. Consequently, the topic provides both basic radiation physics, physical aspects of treatment planning and use of radiation beams. Some knowledge of the effect of ionizing radiation on living tissues is necessary, for those who wish to understand the nature of any treatment using radiation and who also wish to inform patients about such treatment. The topic relates to the effects of radiation on visible structures, in other words, cells and tissues. The radiobiological knowledge described here has been developed in relation to standard radiotherapy. Moreover, the linear quadratic model of cell killing is also applicable for single dose irradiation.

(J Bangladesh Coll Phys Surg 2010; 28: 100-112)

are two types of radiation treatment planning process: forward planning and inverse planning . In inverse treatment planning, an objective function is defined to measure the goodness (quality) of a treatment plan. Two types of objective functions are often used: dose-based model and radiobiological model. The biological model argues that optimization should be based on the biological effects resulting from the underlying dose distributions. The treatment objective is usually to maximize the tumour control probability (TCP) ${ }^{21}$ while maintaining the normal tissue complication probability (NTCP) ${ }^{21}$ to within acceptable levels. Unfortunately, these types of objective function are not rigorously described in the literature and hence it is currently not well suited to optimization approaches. The type of objective function throughout the paper is based solely on dose, in which achieving accurate dose distributions are the main concern.A model has been proposed by Michael C. Ferries, ${ }^{15,15,19}$ Jim-ho Lim and David M. Shepard 15,15,19 in which there are three types of decision variables: 
1. A set of coordinates $\left(\mathrm{x}_{\mathrm{s}}, \mathrm{y}_{\mathrm{s}}, \mathrm{z}_{\mathrm{s}}\right)$ : for each shot of the position centres, each coordinate is a continuous variable to be chosen.

2. A discrete set of collimator sizes: currently four different sizes of focusing helmets are available (4 $\mathrm{mm}, 8 \mathrm{~mm}, 14 \mathrm{~mm}$ and $18 \mathrm{~mm})$; there may be more recent ones.

3. Radiation exposure time: the dose delivered is a linear function of the exposure time; in some suitable sense, one has to determine the optimum time period.

\section{History of Gamma Knife}

The principles of gentle sterile surgery based on knowledge of and respect for the body's compensation mechanisms is part of the mainstream tradition of medical development in the twentieth century. As medical men tend to be conservative, it would take a powerful and courageous intellect to break with such a tradition. Lars Leksell, for many years, Professor of Neurosurgery at the Karolinska Hospital in Stockholm possessed such an intellect. He was one of the most creative neurosurgeons of the $20^{\text {th }}$ century. Over a period from the 1940 to the 1980 's, he devoted his time to method of treatment, which were not confined to taking advantage of the compensation mechanisms that make open surgery possible. On the contrary, his work seems to have had a central aim to reduce operation trauma to an absolute minimum. In his monograph, Stereotaxis and Radiosurgery, he states:

The tools used by the surgeon must be adapted to the task, and where the human brain is concerned they cannot be too refined.

One of Leksell's first clinical contributions ${ }^{1}$ was to device a stereotactic frame for routine use in humans. Prior to this, stereotaxy had been primarily an experimental tool, though a stereotactic technique had been used in the treatment of trigeminal neuralgia and for intracerebral targets. The advantage of the Leksell system $^{1}$ was that it was relatively simple and versatile in operation, when compared with other contemporary stereotactic system. As a result sterotactic surgery gained an impetus which has been maintained to the present day. With Leksell's system, access could be gained to any intracranial region with minimal trauma. However, Leksell's attempt to minimize operative trauma did not stop with the design and further development of a clinical stereotactic system (Gamma knife). ${ }^{1}$ He went further and with a small group of associates devised an apparatus for treating intracranial pathological process, without opening the cranium. This instrument, the Leksell Gamma knife, was designed for use with the Leksell stereotactic system.

\section{Radiosurgery VS Gamma Knife}

The terms radio surgery and Gamma knife have been the source of some controversy. Those who use radio surgical techniques would justify the use of the term as follows. When ionizing radiation is employed to damage or destroy a pathological process, it is vital that normal tissue in the neighbourhood of the lesion remains undamaged. This is achieved in conventional radiotherapy by fractionating the dose and by directing the radiation first from one side and then from the other. The beams are few, broad and seldom more than 8 different beam directions are used; with the gamma knife technique, there are over 200 beams of radiation and they are individually very narrow. This arrangement enables the construction of a very precise radiation field, limited to the pathological lesion. Normal tissue is excluded from dangerous level of irradiation, because in radiosurgery a correlate of the very precise radiation field is a rapid fall in radiation levels just beyond the edge of the lesion. This is due to surgical precision of the radiation field, administered at a single session. That had led to the term radiosurgery. The gamma knife is the first radiosurgery instrument which has gained widespread use in clinical medicine. However, it has no resemblance to any ordinary knife. It is a massive machine, which weighs about eighteen tons. Nonetheless, it delivers an exact field of the radiation. Thus if the technique is called radiosurgery the instrument performing the treatment is by analogy a radiosurgery knife. Since the radiation source is ${ }^{60} \mathrm{Co}$, which emits gamma radiation, this particular radiosurgery instrument is called Gamma knife.

A Leksell Gamma knife treatment has four basic phases:Attaching the head frame, imaging, treatment planning and the treatment itself.

\section{Treatment Goal:}

The plan aims to deliver a high dose of radiation to the intracranial target volume with minimum damage to the surrounding normal tissue. 
1. A complete $50 \%$ iso-dose line coverage of the target volume. This means that the complete target must be covered by a dose that has intensity at least $50 \%$ of the maximum delivered dosage. This can be thought of as a "homogeneity" requirement.

2. To minimize the non-target volume that is covered by a shot or the series of delivered shots. This requirement is clear and can be thought of as a "Conformity" requirement.

3. To limit the amount of dosage that is delivered to certain sensitive structures close to the target. Such requirements can be thought of as "avoidance" requirements.

The number of shots that will be delivered is specified to the optimization tool ${ }^{22}$. While other approaches may try to minimize this number, it is typically straightforward to estimate this number and then develop a plan to optimize other important features for the treatment.

\section{Principles of Stereotaxy}

This technique relates to the location of deep and interaccessible intracerebral structures to a three dimensional Cartesian axis system. The first step in this process $^{7}$ is to enclose the head in such a system. This is done by fixing a rigid metal frame to the head. The borders of the frame then constitute the Cartesian axes, while the cranium serves as a platform to support the frame and the cerebrum is enclosed both physically and conceptually within a microcosm.

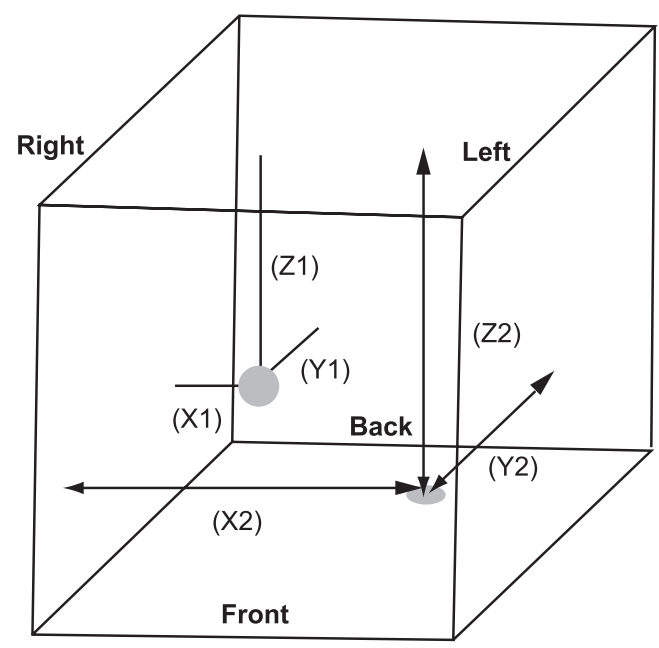

Fig 1: Stereotactic principle (1)
Stereotactic technique relates the position of intracranial targets to visible cranial or extracranial markers. The markers used today consist of a frame which is also a Cartesian axis system. The targets are related to the sides of the frame by perpendiculars dropped from the frame to the target. As can be seen, only one point is identified by the values $\mathrm{X} 1, \mathrm{Y} 1, \mathrm{Z} 1$ just as only one other and quite distinct point is identified by the values X2,Y2,Z2. Thus any intracranial location can easily be identified in relation to the frame which is fixed to the head. The orientation of the frame is a secondary consideration. It is the frame and not the head which is used to localize. This is useful in Gamma knife work where it may be convenient to place the frame eccentrically or to rotate it in respect of the head.

Where every point can be precisely defined in space. The way in which a point within the frame is defined in terms of the three Cartesian axes is depicted in Fig.1. Thus it is possible to define any intracranial target in respect of the frame. However, to be of any use the frame must itself be a platform for a device, which will hold an instrument or electrode, to be introduced into the brain, to reach the target. The small diameter of the instruments used and the mechanical stability with which they are held and introduced, by means of a rigid holder and guide, and the extreme accuracy of the localization implicit in the method are the basis of the exceptionally atraumatic nature of stereotactic procedures.

\section{The Leksell System ${ }^{1}$}

A great variety of different stereotactic systems have been designed over the last forty years. Each system has its protagonists and its special fields of application. For a description of the essential technical principles of stereotactic surgery reference will be made to the Leksell system, because it is the system used in Gamma knife surgery. These principles are on the whole independent of the system used, though the way in which technical problems are solved differs from system to system. The sides of the cubic frame constitute the axes of the instrument. An arc is mounted on the instrument in an adjustable holder which is regulated in respect of the desired values in the three axes. The arc may be rotated backwards and forwards, with respect to the frame. The instrument holder is mounted on the arc and may be moved transversely across the whole circumference of the arc. When the axis values for the target point have been determined, the centre of the arc will always 
coincide with the target point. The arrangement allows a needle to be pointed as its target from an almost infinite number of directions. Thus, it is simple to design an optimal trajectory for the instrument to be introduced into the depths of the brain, avoiding especially sensitive structures, for example eloquent brain or important blood vessels. An important point of the design is that, for a given target setting, the point of the instrument introduced to the centre of the arc does not move. It does not move irrespective of how the direction of the shaft of the instrument is varied, by moving the arc backwards and forwards or by moving the needle, the holder transversely across the arc. This effect is quite uncanny and is illustrated in Fig 2

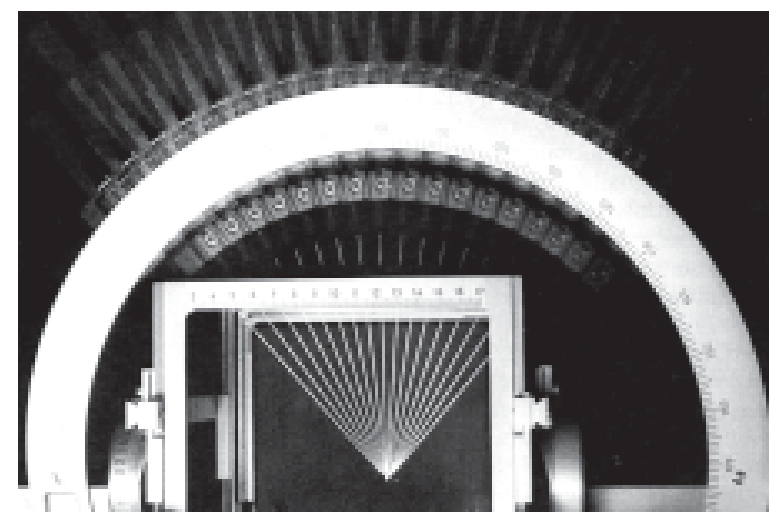

Fig 2: Stereotactic principle (2)

This is an illustration of a stereotactic frame, with a needle mounted, as it would be for penetration to a specified intracranial target. The picture is retaken with the needle approaching the target from a variety of directions. As can be clearly seen, the direction of the needle in no way affects the positioning of the point, which is placed at the desired target, as determined by appropriate adjustment of the various axes of the system.

The name of the axes are of course $\mathrm{X}$-axis, Y-axis, and Z-axis. However the direction of the disparate axes varies from stereotactic system to stereotactic system. The choice of which axis points in which direction was originally arbitrary. However, with the Leksell system, as with most others in use today, the convention has become that the $\mathrm{X}$-axis runs from side to side, the $\mathrm{Y}$ axis runs from behind forwards and the $\mathrm{Z}$-axis runs from above downwards.

For anyone who wishes to remember the direction of the axes, the common zero point is above and behind the right ear.

\section{Target identification}

The features of a stereotactic system, which have been outlined so far describe how a point in space, a so-called target point may be defined in terms of the reference axes system, built into the stereotactic frame, fixed to the head. If the target is "visible" for example a space occupying lesion, then a general knowledge of cerebral anatomy together with adequate imaging techniques will suffice. However, in the early days, stereotactic technique was almost exclusively used for the treatment of functional disorders. The targets in this situation are "invisible" consisting of discrete nuclei or tracts within larger anatomical entities, such as the thalamus or the basal ganglia. To locate such targets, a map of the region is required or rather a collection of maps in an atlas. An atlas of the internal cerebral anatomy of a variety of laboratory animals has already been produced by Horsley and Clarke in the first decade of the $20^{\text {th }}$ century. The production of a human stereotactic atlas in 1952 was one of the major contributions of the pioneers Spiegel and Wycis, mentioned above.

\section{Newer Localization Methods.}

It is interesting, that Leksell's first stereotactic operation on a patient, using his own instrument, was to instill radioactive isotopes, into a craniopharyngioma cyst; the development of computer assisted tomography (CT) and more recently magnetic resonance imaging (MRI) have greatly

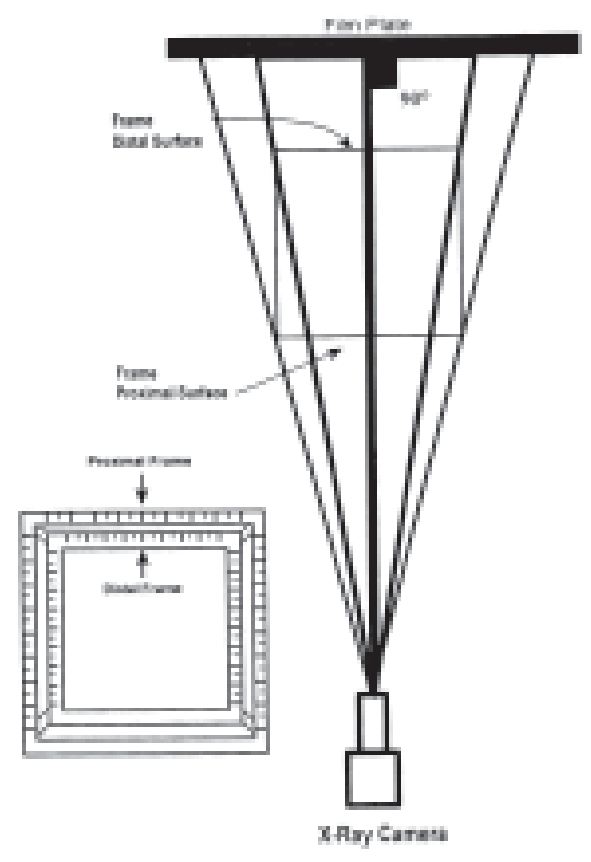

Fig 3: Angiogram Indicator 
facilitated stereotactic procedures. These techniques render space-occupying lesions truly visible and thus simple to localize. Moreover, the modern machines can incorporate software which will superimpose a stereotactic grid on the film and this makes localization extremely simple.

This use of the stereotactic frame, with CT and MRI units necessitated the development of an adaptor to fix the stereotactic frame to the CT/MRI table, to ensure that the axis system of the frame and the axis system of CT/MRI software were concordant. A word of caution must be mentioned in respect of using MRI for target localization. There is a degree of anatomical distortion in MRI pictures, which increases from the centre to the edges. This distortion must be minimized by repeated checks. It makes central placement of the target within the frame even more important than otherwise; a topic to which we shall return.

\section{Modern Indications for Stereotaxy}

Stereotactic technique ${ }^{1}$, following the advent of CT imaging has a large number of indications, most of which have been mentioned. There has however been a tendency for its use to be restricted to a relatively small number of enthusiasts. It is not, even today, used routinely by all neurosurgeons. This is partly because, in the majority of centres, it has been used for functional work, which requires a specialized neurophysiological knowledge, that is not a part of all general neurosurgical training programmes. Furthermore, the basis of the technique is not technical surgical virtuosity but rather the avoidance of the need for such virtuosity. The Karolinska Hospital Neurosurgery Department, under Leksell's aegis taught that stereotaxy was not to be considered an alternative to other forms of treatment but to be used in addition to the more traditional armamentarium of neurosurgical options. Any method which reduces the per-operative trauma of a neurosurgical procedure to a minimum must be considered as preferable to one relying on surgical virtuosity, which takes advantage of the brain's compensation mechanisms. There are then three main areas of indication, which have been used for open stereotactic procedures. Firstly, there is the original group of functional illnesses already mentioned. Secondly, there are the direct primary treatments of cystic space occupying lesions, for example the installation of radioactive isotopes into craniopharyngioma cysts and the aspiration of abscesses. Moreover, aspiration of intracerebral haematomas may also be performed stereotactically, though the timing and indications remain a matter for debate. Finally, solid tumours may be biopsied as a preliminary to rational treatment planning.

\section{Combination of target and radiation}

Open stereotactic technique ${ }^{1}$ in the 1990 's was essential for the treatment of a number of functional conditions and cystic space occupying lesions. It has an important part to play in the investigation of tumours and can help to increase the number which are accessible to treatment. It is regrettable that the technique is not, even today, in routine use in all Neurosurgical Departments. This is undoubtedly the result of a misunderstanding of the role of stereotactic technique for a modern Neurosurgical service. It may also be the result of the innate conservatism of those who finance the acquisition of the necessary equipment and of those who would have to use it, once acquired. In the opinion of the researchers, the increase in precision and the reduction of surgical trauma, inherent in the technique makes it very difficult to argue against its widespread, routine application. Nonetheless, it has its limitations. As yet it cannot of itself be used in the effective removal of solid tumours. However, it can be employed to guide not only solid instruments but also ionizing irradiation to mass lesion targets. It is just this combination of stereotactic guidance and narrow beam, high-energy radiation, to a precisely defined target, that is the basis of Gamma knife Radiosurgery.

\section{Dose Distribution Model}

In this proposed method the dose distribution ${ }^{9,18,22}$ is modeled non-linearly and a smoothing continuous approach used to treat discrete problem choice. The resulting nonlinear programme is not convex and several heuristic approaches are used to improve solution time and quality. 
Gamma knife radiosurgery begins (after administering local anesthesia) by fixing a stereotactic coordinate head frame to the patient head using adjustable posts and fixation screws. Steretactic technique relates the position of intracranial target to visible cranial or extracranial markers. This principle fully depends on three dimensional coordinate system, second is the Leksell system(Leksell head frame); this is an illustration of a stereotactic frame, with a needle mounted, as it would be for penetration to a specified intracranial target.

We require an algebraic model of the distribution of the dose for use in our optimization formulations. In this model, we let $\mathrm{S}$ represent the set of the shots that we will consider, and $\mathrm{W}$ represent the possible shot sizes (typically $4 \mathrm{~mm}, 8 \mathrm{~mm}, 14 \mathrm{~mm}$ and $18 \mathrm{~mm}$ ). The complete dose distribution can be calculated as a sum of contributions from each shot delivered, once the location of the centre of the shot $\left(\mathrm{x}_{\mathrm{s}}, \mathrm{y}_{\mathrm{s}}, \mathrm{z}_{\mathrm{s}}\right)$ is known, and the length of time of delivery $t_{\mathrm{s}, \mathrm{w}}$ is known. In practice this means that for all $(\mathrm{i}, \mathrm{j}, \mathrm{k})$

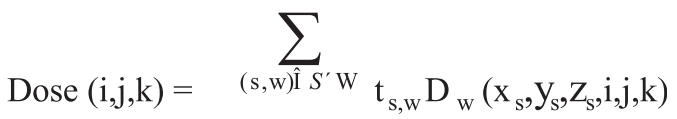

where $\mathrm{D}_{\mathrm{w}}\left(\mathrm{x}_{\mathrm{s}}, \mathrm{y}_{\mathrm{s}}, \mathrm{z}_{\mathrm{s}}, \mathrm{i}, \mathrm{j}, \mathrm{k}\right)$ is the dose delivered to the $\operatorname{voxel}(\mathrm{i}, \mathrm{j}, \mathrm{k})$ by the shot of width $\mathrm{w}$ centered at $\left(\mathrm{x}_{\mathrm{s}}, \mathrm{y}_{\mathrm{s}}, \mathrm{z}_{\mathrm{s}}\right)$.

To determine the form of $\mathrm{D}_{\mathrm{w}}$, the following procedure was followed.

$D_{(d)}=\frac{D(0,0,0, d, 0,0)+D(0,0,0,0, d, 0)+D(0,0,0,0,0, d)}{3}$

These values were used as data in a nonlinear parameter estimation problem.

The problem is thus reduced to determining a functional form for the dose delivered at a voxel $(\mathrm{i}, \mathrm{j}, \mathrm{k})$ from the shot centered at $\left(\mathrm{x}_{\mathrm{s}}, \mathrm{y}_{\mathrm{s}}, \mathrm{z}_{\mathrm{s}}\right)$

We therefore used the following functional form

$\mathrm{D}_{\mathrm{w}}\left(\mathrm{x}_{\mathrm{s}} \mathrm{Y}_{\mathrm{s}}, \mathrm{Z}_{s}, \mathrm{j}, \mathrm{j}, \mathrm{k}\right)=\sum_{p=1}^{2}{ }_{p}\left(1-\operatorname{erf}\left(\frac{\left.\sqrt{\left\{\left(i-x_{s}\right)^{2}+{ }_{p}^{y}\left(j-y_{s}\right)^{2}+{ }_{p}^{2}\left(k-z_{s}\right)^{2}\right.}\right\}-{ }_{p}}{\sigma_{p}}\right)\right)$

and fit the ten parameters $\lambda_{p}, \mu_{p}^{y}, \mu_{p}^{z}, \gamma_{p}$ and $\sigma_{p}$; $\mathrm{p}=1,2$, to the data described above via least squares, with different values for each shot width. The notation erf(x) represents the integral of the standard normal distribution from - " to $\mathrm{x}$.
The resulting nonlinear optimization problem ${ }^{3,14,18}$

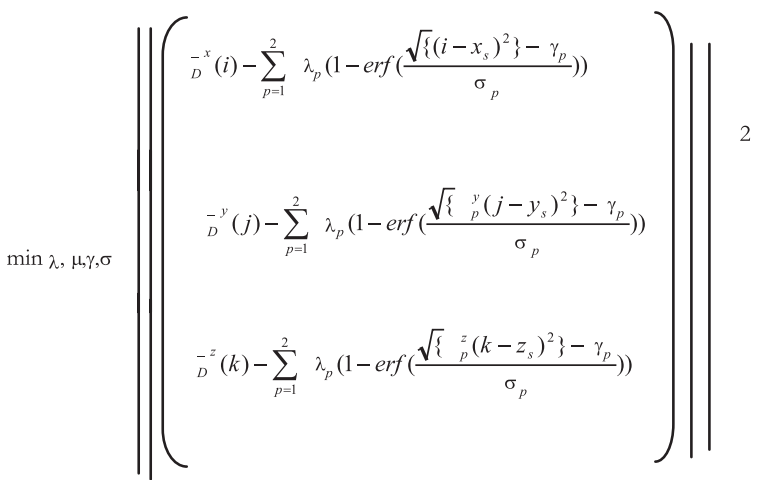

was solved using CONOPT. ${ }^{3,4}$

\section{Dose Computation}

To ensure the precise localization of the target volume, the Leksell head frame was used. The standard procedure for attaching a stereotactic frame to the patient's head was followed so that the 3D reference points could be seen on computed tomography (CT) and magnetic resonance imaging (MRI) scans. In some cases, this delineation was guided by angiography. After transferring the images to a computer, the cranial surface contours on slice images could be automatically detected. The target volume and critical tissues on these images were determined by a physicianphysicist team to ensure reliability or identify any special clinical considerations. Then, based on the external skull contours and target contours, we calculated the different source-skin distances necessary.

To optimize the irradiation parameters, we established a mathematical model, in which dose computation played an important role. We used the same notation as Yan and coworkers ${ }^{11}$ used for a gamma knife treatment system; the dose at one point $\mathrm{p}$ given by one static gamma ray source can be written as:

$\mathrm{D}(\mathrm{d}, \mathrm{r}, \mathrm{s})=\mathrm{M} \cdot \mathrm{OF}(\mathrm{s})$. TMR $(\mathrm{d}, \mathrm{s}) . \mathrm{OAR}(\mathrm{d}, \mathrm{r}, \mathrm{s}) \cdot \mathrm{F}^{2}$,

Where, TMR is the tissue maximum ratio;

OAR is the off axis ratio

$\mathrm{F}^{2}$ is the inverse correction factor

$\mathrm{OF}$ is the output factor

$d$ is the distance of the surface of the scalp and the point of calculation of the central axis along a ray from the source to the shot.

$r$ is the radial distance from the central axis to the point of calculation.

$\mathrm{s}$ is the size of the collimator.

$\mathrm{M}$ is the converting dose to the source irradiation time. 


\section{Nonlinear Optimization}

Once a description of the dose is determined, the optimization model ${ }^{14,18}$ can be formulated. The basic variables of the optimization we consider include the co-ordinates of the center location of the shot $\left(\mathrm{x}_{\mathrm{s}}, \mathrm{y}_{\mathrm{s}}, \mathrm{z}_{\mathrm{s}}\right)$, the width of the shot $\mathrm{w}$, and the time $\mathrm{t}_{\mathrm{s}, \mathrm{w}}$ that each shot is exposed. In practice, we consider a grid $\mathrm{G}$ of voxels. There are two types of voxels : T represents the subset of voxels that are within the target and $\mathrm{N}$ represents the subset of voxels that are out of the target.

The 50\% isodose curve is a curve that encompasses all of the voxels that receive at least $50 \%$ of that maximum dose that is delivered to any voxel in the patient. We model such a constraint by imposing strict lower and upper bounds on the dose allowed in the target, namely for all (i, j, k) ${ }^{\circ} \mathrm{T}$

$$
\theta \leq \text { Dose }(\mathrm{i}, \mathrm{j}, \mathrm{k}) \leq 1
$$

In this way, the 100 è \% isodose curve is guaranteed to cover the target.

A shot width $\mathrm{w}$ is used at location $\mathrm{s}$ if $\mathrm{ts}, \mathrm{w}>0$. The main idea is to approximate step function

$$
\begin{gathered}
\mathrm{H}(\mathrm{t})=\left\{\begin{array}{c}
1 \text { if } \\
0
\end{array} \text { if } \quad t>0\right. \\
\text { by a non linear function, } \mathrm{H}(\mathrm{t}) \approx \mathrm{H} \alpha(\mathrm{t}):=\frac{2 \arctan (\quad \alpha t)}{\prod}
\end{gathered}
$$

For increasing values of, $\mathrm{H}$ becomes a closer approximation to the step function $\mathrm{H}$. This process is typically called smoothing. The set of shot widths for a given number of shots ' $n$ ' is chosen by imposing the constraint

$$
\mathrm{n}=\sum_{(s, w) \in\{1, \ldots n\} \times W} H \alpha\left(t_{s}, \mathrm{w}\right)
$$

we solve the following problem :

$$
\begin{array}{r}
\sum_{\operatorname{Min}(i, j, k) \in N} \operatorname{Dose}(i, j, k) \\
\text { Subject to } \\
\text { Dose }(\mathrm{i}, \mathrm{j}, \mathrm{k},)=\sum_{(s, w) \in S \times W} t_{s},{ }_{w} D_{w}\left(x_{s}, y_{s}, z_{s}, i, j, k\right) \\
\theta \leq \operatorname{Dose}(i, j, k) \leq 1, \forall(i, j, k) \in T \\
\mathrm{n}=\sum_{(s, w) \in\{1, \ldots, n\} \times W} H\left(\begin{array}{ll}
t_{s}, \\
\mathrm{t}_{\mathrm{s}, \mathrm{w}} \geq 0
\end{array}\right.
\end{array}
$$

note that both problems are highly non convex so there is no guaranty of global optimality

\section{Radio Physics}

\section{Basic Concepts}

The basic mechanisms, underlying the effect of radiation on matter occur at the atomic and subatomic level. The concept of an atom, consisting of a nucleus containing a specific number of positively charged protons and noncharged neutrons, surrounded by orbits of electrons is familiar. Another important set of concepts in the study of atoms and subatomic particles are embodied in theory; Quantum theory. Quantum theory was developed to explain finding which indicated that electromagnetic radiation sometimes appeared to behave as a wave and sometimes appeared to behave as a stream of particles or quanta, each carrying a certain specific amount of energy, defined by Planck Radiation formula:

$$
\mathrm{E}=\mathrm{hv}
$$

where $\mathrm{E}$ is energy, $\mathrm{h}$ is Planck's constant and $\mathrm{v}$ is the frequency of the radiation. Moreover, it is also true that subatomic particles may be considered to have wave like properties. Another important concept, for the understanding of radiations interaction with matter, is that energy and matter are interconvertable, accordingly to Einstein's famous equation:

$$
\mathrm{E}=\mathrm{mc}^{2}
$$

where $\mathrm{E}$ is energy, $\mathrm{m}$ is the mass of particle being converted into or arising from energy and $c$ is the velocity of light. This dual nature of radiation and subatomic particles is not intuitive and has been considered difficult to understand. However, it is not really so. Many familiar objects have different properties depending on how they are observed. Irrespective of whether waves or particles are considered, an atom can only emit or absorb energy in discrete, discontinuous quanta. The quanta so emitted or absorbed will have a particular energy and by the same token a particular wavelength; in accordance with Planck's equation.

The term ionising radiation refers to radiation which has a sufficiently high energy to be able to dislodge electrons from atoms, or disrupt the bonds between atoms and molecules. Examples of this sort of radiation are ultraviolet ray, X-rays and gamma rays. An atom deprived of an electron will have a net positive charge and thus will have become an ion hence the term ionising radiation. 
There are two sorts of radiation source used in radiation treatment, artificially generated irradiation from manmade machines and spontaneously generated radiation from radio-nuclides.

There are two basic kinds of radiation in current use. Electromagnetic radiation has no mass and travels at the velocity of $\operatorname{light}\left(\mathrm{c}=310^{8} \mathrm{~m} / \mathrm{s}\right)$. Particle radiation consisting of for examples protons, neutrons or electrons, has mass and travels at a lesser velocity. Both particles and electromagnetic radiation lose energy to matter by interacting with it. If a radiation passes through matter without striking an atom no ionisation will occur.

\section{Electromagnetic Radiation.}

There is of course a vast range of electromagnetic radiation from the lowest frequency radio waves (frequency $10 \mathrm{kHz}$, wavelength 30 kilometers) up to cosmic rays (frequency $10^{24} \mathrm{KHz}$, wavelength $1 / 1000$ millionth of an Angstrom unit). In low frequency, long wavelength radiation the wave-like properties dominate. In high frequency, short wavelength radiation, the particle-like properties dominate. For the present purpose, the range of interest is X-rays (approximate $10^{15}$ to $10^{21} \mathrm{~Hz}$.) and gamma rays (approximate, $10^{18}$ to $10^{24} \mathrm{~Hz}$ ).

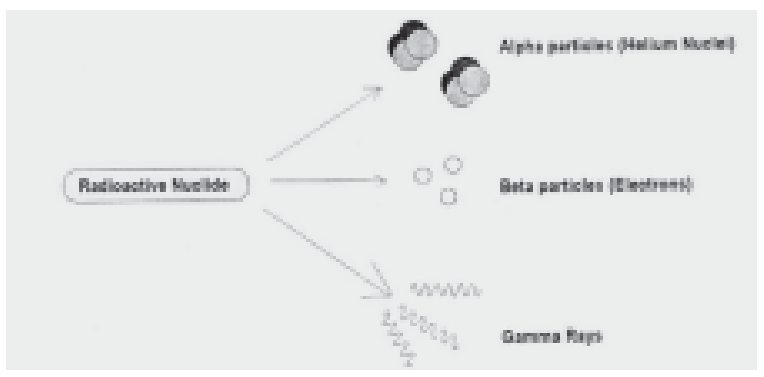

Fig. 4 Radioactive Decay

There are three main types of radioactive breakdown, producing three main products as illustrated here. However, not all radioactive breakdowns produce all products. Thus alpha particles are mostly produced by isotopes of high atomic weight, for example Uranium. Cobalt-60 produces gamma waves, but also loses electrons.

As electromagnetic radiation, gamma rays are produced in a different way (Fig 4) when the nucleus of an atom is in an excited state it can decay to a stable state by emission of one or more photons, (quanta a electromagnetic energy) called in this case gamma rays or gamma photons. The other products of radioactive breakdown are alpha particles (helium nuclei with 2 protons and 2 neutrons) and beta particles (electron) and neutrons. Alpha particles produced by radioactive transformation have too low a penetration to be of much use in clinical practice. Beta radiation also has a low penetration but it may be used following implantation of isotopes in tissue. One example, in the field of neurosurgery is the highly successful treatment of craniopharyngioma cysts with instillation of radioactive yttrium, which emits pure beta radiation. This is not unusual. Many nuclides emit a greater proportion of one of the products of radioactive breakdown than of the others. Thus Uranium-235 is primarily an alpha emitter, just as coblt-60 is mainly a gamma emitter but also emits beta particles.

\section{Particle Radiation.}

Electrons and protons are the most commonly used particles in current therapeutic use. An adapted linear accelerator or a betatron, a special sort of accelerator can produce electron beams. Commonest energies of electron beams are 7 to $18 \mathrm{MeV}$, for the linear accelerator and 12.4 to $124 \mathrm{MeV}$ for a betatron.

Proton particles are produced in particle accelerators, such as the synchrocyclotron. Particle beams have special characteristics, enabling the delivery of a sharply defined dose deep in the tissues, with relative sparing of the tissues on the way in to the high dose volume. The energies of those current use ranges from 72 to 100 $\mathrm{MeV}$.

The Effect of Electromagnetic Radiation on Matter Ionisation is a chemical as well as physical change. Electromagnetic radiations can react with matter in a variety of ways; for example reflection, refraction interference, that is different forms of scattering. They can induce chemical change only by absorption. When ionising radiation is absorbed it interacts with atoms to detach electrons from their orbits. The energy of these electrons is part of the energy of the incoming photons. There are three main ways in which such interactions between radiation and matter occur, depending on the energy of the radiation. Finally, it should be repeated that some radiation will go through whatever matter is being irradiated without interacting with it. 


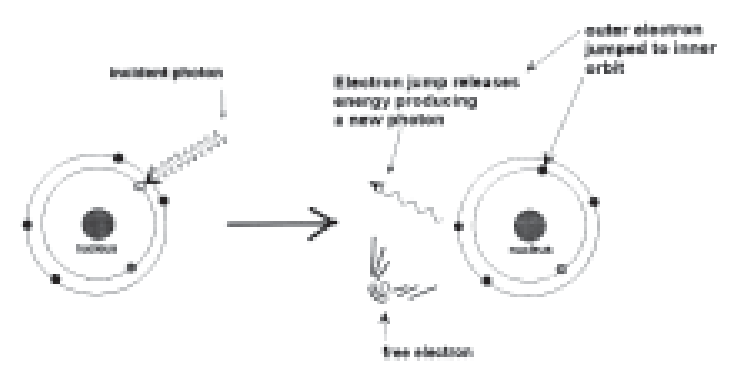

Wevirs than

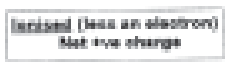

Fig. 5 Photoelectric Effect

The photoelectric effect was discovered following the observation that when a spark appears at a gap between two electrodes its appearance could be facilitated by shining light at the gap. The spark was due to electrons crossing the gap. The photoelectric effect is due to the incoming photon from the light loosening an electron and thus facilitating the generation of a spark. The electron released is from the inner shell. The energy of the ongoing photon is discharged when an electron jumps from an outer to the inner electron shell. The energy of this photon is equal to the energy of the incoming photon less the energy imparted to the free electron. Note that the frequency of the incoming photon is less than that of the ongoing photon. This reflects the relationship between the energy and frequency of a photon.

The nuclei of atoms can only be affected by gamma radiation, not by ultra-violet radiation or X-rays; only gamma rays have enough energy to penetrate a nucleus.

\section{The Photo Electric Effect.}

This is the major energy absorption mechanism for low energy $\mathrm{X}$-ray beams up to $50 \mathrm{keV}$, though it also occurs at higher energy levels. All the energy of a given photon is absorbed in detaching an electron from one of the inmost shells of an atom. An outer electron will then hop into the insufficiently filled inner shell, resulting in a change in energy level and the emission of a photon. of X-rays (fig5). The kinetic energy of the originally ejected inner electron will be equal to the energy of the incident photon minus the energy required to detach it from its orbit.

\section{Compton Scattering}

With higher energy X-ray beams and gamma rays, with an energy between approximately $90 \mathrm{kev}$ and $5 \mathrm{MeV}$, a different effect occurs; involving the interaction of the radiation photon with electrons in the outer shell of the atom. Some of the photon's energy will be dissipated in detaching the electron from its path and in giving it kinetic energy. The rest of the energy will continue as a new photon with an energy equal to the energy of the incident photon less the energy required to detech the electron and the kinetic energy delivered to that electron (fig 6). This new photon with a lower energy will naturally have a longer wave length. Quantitative analysis of the effects of radiation in water has shown that the vast majority of the energy absorbed is related to the detached electrons and not to the ongoing lower energy photons.

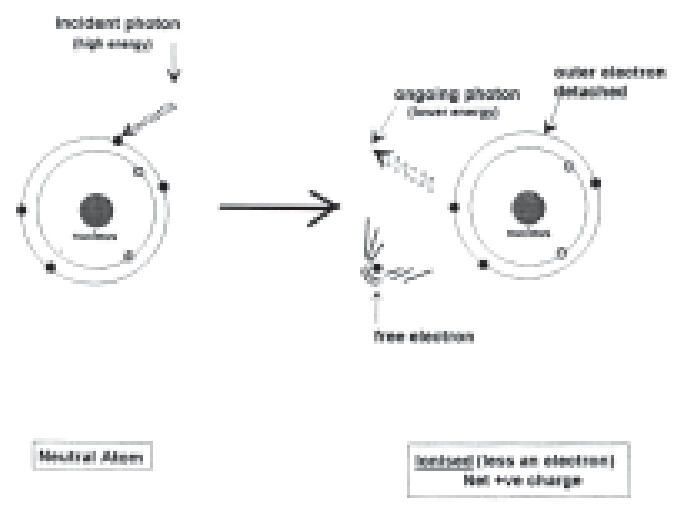

Fig. 6 Compton Scattering

Compton found that when X-rays are dispersed in a crystal, there was a change in the frequency of the $\mathrm{X}$ rays, indicating a loss of energy. At the same time an outer electron is freed. The lower frequency of the ongoing $\mathrm{X}$-rays is equal to the energy of the incoming $\mathrm{X}$-ray photons less the energy imparted to the ongoing electron. Note that, as in the photoelectric effect, there is a net gain of one positive charge, and the atom is ionized. This time the energy liberated comes directly from the incoming photon. It is not mediated by means of an electron hopping from one orbit to another. It is the most likely process to be responsible for ionization during Gamma Knife surgery.

\section{Pair Production.}

When a photon passes close to the nucleus of an atom it is exposed to the powerful energy field around that nucleus and may thus be converted from a photon of energy into matter, in the form of a pair of electrons (or electron-positron pair ). Since the mass of an electron is equivalent to $0.511 \mathrm{MeV}$ the energy of the incident photon must be at least $0.511 \mathrm{x} 2$ or $1.022 \mathrm{MeV}$. One of the pair of electrons has a positive charge(positron) and the other a negative charge(electron). Both these 
electrons pass through the absorbing matter exciting and ionising atoms (fig 7).

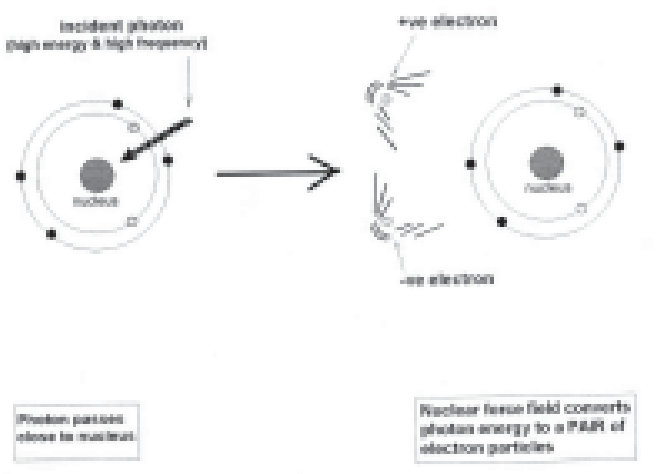

Fig. 7 Pair Production

In this case, the incoming photon has a sufficiently high energy to reach close to the nucleus. Here it is affected by the powerful field and is converted into energy. Two electrons are formed in accordance with the law of conservation of electric charge. Thus the minimum number of electrons that can be formed in this way is one with a positive and one with a negative charge. In this way electric neutrality is maintained, the law is obeyed.

The different mechanisms of energy absorption are not mutually exclusive: though the coexiestense of the photoelectric effect and pair production is not thought to occur. However, the radiation energy range, associated with compton scattering occurring at its lower end is associated with the photoelectric effects, while at its upper range it will be associated with pair production.

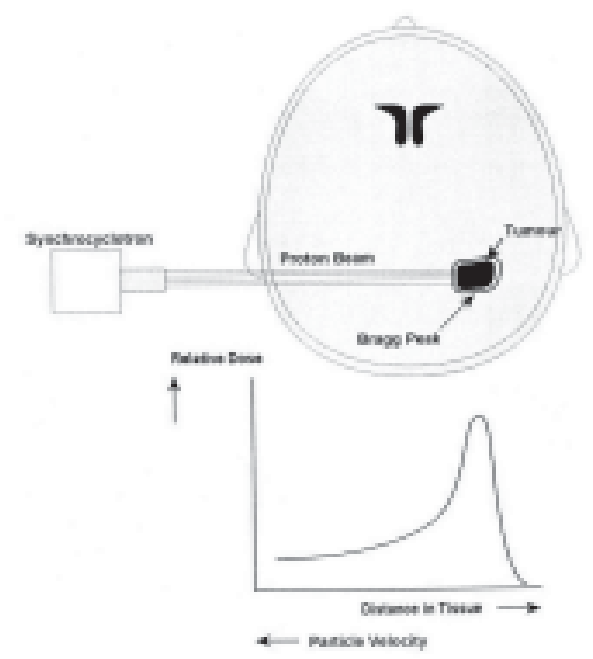

Fig 8 Focal Radiation (Bragg Peak) Most radiation energy delivered from particle radiation is lost when the particles decelerate. This deceleration of particles enables the concentration of the radiation dose over a tightly controlled sharply defined volume, called the Bragg Peak. This is one of the best known techniques for delivering focused radiation today. For the sake of presenting the picture of the radiation and the graph of particle deceleration concordantly; the source in this diagram is placed on the opposite side from the lesion.

\section{The Effects of Charged Particles on Matter.}

A crucial difference in the pattern of energy absorption between particles and electromagnetic radiation is related to this characteristic of particles: that they can decelerate while radiations are bound to travel at the speed of light.

The absorption of energy associated with the passage of particles through matter is described by the Linear Energy Transfer(LET), described by the formula - $\mathrm{dE} /$ $\mathrm{dx}$ where $\mathrm{dE}$ is energy loss and $\mathrm{dx}$ is unit distance travelled. The units of $\mathrm{LET}$ are $\mathrm{KeV} / \mathrm{mu}$ : where $1 \mathrm{mu}=$ $10^{-6} \mathrm{~m}$. The energy loss of particle is reflected by ionisations along the course of its passage. How far the particles will travel in a medium- such as a living tissueis a function of the density of the medium and its atomic weight on the one hand, and the mass and the velocity of the particle on the other. Protons as an example of "charged heavy particles", with their greater mass can penetrate more deeply.

There is relatively little energy loss along the track of a proton beam, so long as the particle is moving quickly. Thus, such heavy particles have a low LET in the part of their tracks where they are moving fast. However, more and more of their energy is absorbed as they decelerate, so that this part of the track has a high LET. Since most energy absorption occurs at the distal end of the track, most of the ionisations also occur in this region. A consequence of this phenomenon is that particles like protons, with an appropriate delivery system can be used to produce very precisely defined radiation fields at specific distances from the particle source. The precisely defined area of intense irradiation at the end of a low LET track following the passage of protons, is called a "Bragg Peak". However, taking advantage of the Bragg Peak phenomenon is not the only way in which a proton beam may be used to produce a well localized volume of high radiation energy delivery. Cross firing of a number of narrow proton beams will also produce a region of high dose where the beams cross, while the amount of dose delivered along the beam outside the cross firing region will be 
low because of proton radiation's low LET. To avoid the development of a Bragg Peak a proton source is used with a high energy and therefore a high penetration, so that the deceleration of protons, necessary for a Bragg Peak will occur after the protons have passed through the living tissue and emerged on the far side (Fig,6.5). Obtaining a sharply defined radiation dose, by using cross firing of a number of narrow radiation beams is central principle of Gamma knife radiosurgery.

\section{Radio Physics and The Gamma Knife}

Gamma radiation is of course non-particulate electromagnetic radiation, to be considered either as waves or photons. The gamma radiation produced by ${ }^{60} \mathrm{Co}$ has two energies, reflecting two distinct radioactive breakdown path ways. The gamma radiation from these two reaction series has an energy of either 1.17 or 1.33 $\mathrm{MeV}$ depending on which radioactive breakdown pathway is being considered. With radiation energy within this range, according to the description on earlier, most of the interaction between radiation and irradiated tissue can be expected to be mediated by compton scattering and to a lesser degree pair production. The energy level of this radiation is sufficient to give it a high power of penetration. It has a low linear energy transfer (LET). The narrow beams, essential to the technique, are produced by a construction which forces the radiation through collimators in the form of small metal tubes, mounted in the machine's helmet. The size of the collimators is defined in terms of the diameter of the $50 \%$ isodose around the centre of the target. These collimator sizes are $4 \mathrm{~mm}, 8 \mathrm{~mm}, 14 \mathrm{~mm}$ and $18 \mathrm{~mm}$.

\section{Radiochemistry}

\section{Early Effects}

Radiophysics describes direct effect of radiation, which take place within fraction of microsecond. A common feature of all forms of radiation is the production of free electrons at speed through absorbent medium. These can combine with ions of the same sort and also combine with atoms of other molecules, producing energized unstable products. This is the basis of the indirect effect. In living tissues, among the available molecules which can thus react with electrons is the water molecule, which is present in abundance. In a matter of microseconds free radicals can form according to , for example ,the following equations .

$$
\begin{aligned}
& \mathrm{H}_{2} \mathrm{O} \mathrm{H}_{2} \mathrm{O}^{+}+\mathrm{e}^{-} \\
& \mathrm{H}_{2} \mathrm{O}^{+} \mathrm{H}^{+}+\mathrm{OH} \\
& \mathrm{H}_{2} \mathrm{O}^{+}+\mathrm{e}^{-} \mathrm{H}^{+}+\mathrm{OH}^{-}
\end{aligned}
$$

\section{Radiation Injuries of Nucleic Acid Molecules}

Chemical bonds may be broken, polymers may depolymerize or new unphysiological polymerization may occur. These processes may be reversible and be subsequently repaired, but they may be permanent leading to biochemical injury. The biochemical changes occur over seconds to hours following irradiation. It is generally thought that the most important target for biochemical injury is the hereditary molecule DNA. There are three main lines of evidence. Firstly, selective irradiation of nuclei and cytoplasm separately has shown that the nucleus is far more susceptible. Secondly, cell death is more easily achieved, when binding radioactive nuclides to different intracellular macromolecules, if the said nuclides are incorporated into the DNA. Finally, there is a strong correlation between the radiation responsiveness of a cell and its DNA content. Thus DNA molecules tend to be fragile. There are four major sort of DNA injuries which are considered to be the most common cause of radiation. These are base damage, cross links with nuclear protein, single DNA strand breaks-SSB -or double DNA strand breaks -DSB (Fig.9). The evidence suggests that it is those DSB, which do not repair after several hours, which are responsible for the sterilization of the cell. It would seem that $1 \mathrm{~Gy}$ of radiation produces about 2000 initial single strand breaks and about 40 Double strand breaks. However, the lesion that counts is the DSB which is not repaired.
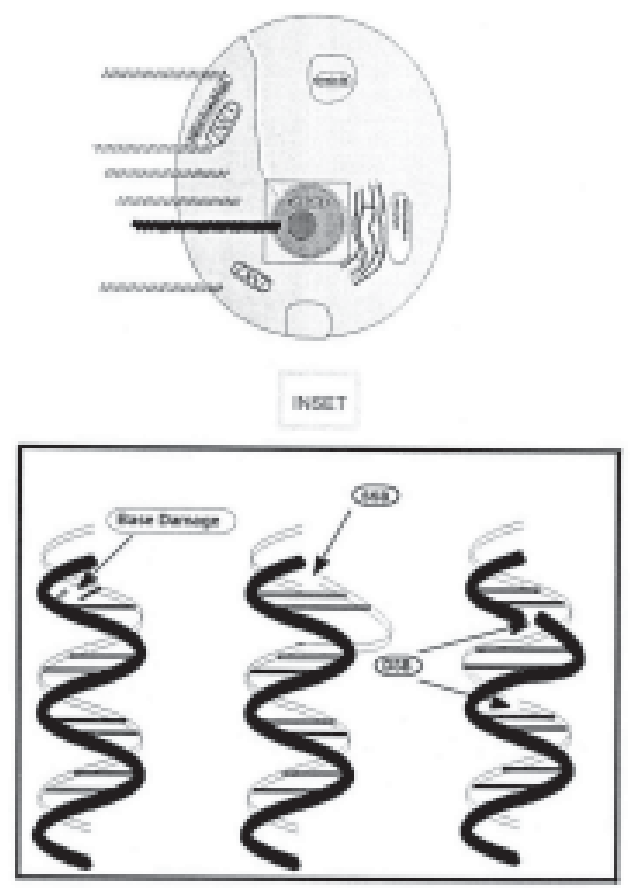

Fig.9. Cellular Radiation Targets 
Radiation can strike anywhere in a cell, but nuclear DNA is seen as the most important target, as indicated here by the thicker radiation penetrating the nucleus. The inset shows 3 of the common forms of DNA damage. About 2000 single strand breaks (SSBs) occur for 1 Gy, which at the same time produces about 40 double strand breaks (DSBs). It is DSBs which are the lethal lesion. But $1 \mathrm{~Gy}$ is also considered to produce an average of 1 lethal lesion per cell, indicating the efficiency of the repair processes.

\section{Biological Changes.}

Ionising radiation can damage living tissue, physically and chemically, at the subatomic and molecular level. These chemical and physical changes will be expressed biologically in two main ways. The membranes, enzymes and protein factory of the affected cells may cease to function or function in a deranged fashion. The reproductive functions of the cell may be damaged with destruction or damage to chromosomes, delay in mitosis, mutation and change in the cell cycle. Reflecting the possibility for repair at the physical and chemical levels, these biological changes may recover or they may not. Finally, if the above changes are not lethal in the short term, over a time scale of month to years late effects may be seen in the form of premature aging, carcinogenesis or growth disturbances in the young.

\section{Radiobiology}

\section{Cell Survival Studies.}

Cell death is the loss of the capacity for indefinite proliferation. Cell survival ${ }^{1}$ studies consists in saving the cells from death after the effect of radiation; which is in vitro, in other words. The proportion of surviving cells is called survival fraction and is a much used quantitative indicator of the effect of the radiation. The type of statistics used in calculating the chance of cell kill are called Poisson Statistics. It is estimated that 1 Gy of radiation will give rise to $10^{5}$ ionisation per cell. On the other hand this amount of radiation produces only about 40 double strand breaks in the cell's DNA. Using poisson statistic it is calculated that the percentage of cells which survive, when a radiation dose, sufficient to produce an average on lethal lesion per cell is delivered, is $\mathrm{e}^{-1}$ or $37 \%$. This is called the survival fraction. The dose producing a survival fraction of $\mathrm{e}^{-1}$ is called $\mathrm{D}_{0}$.
The relationship between the survival fraction and the dose can be expressed as an equation.

The linear quadratic equation has the form

$\mathrm{p}=\mathrm{e}^{-(\mathrm{D} 2)} \quad$ where $\mathrm{p}$ is the survival fraction, alpa and beta are constants, linear and quadratic components of the equation.

There are two main conceptual models which, it is postulated may explain the form of the equation. The first is called the Lethal-Potentially-Lethal model (Fig 10).. The second model is called the Saturation Repair Model (Fig 11).

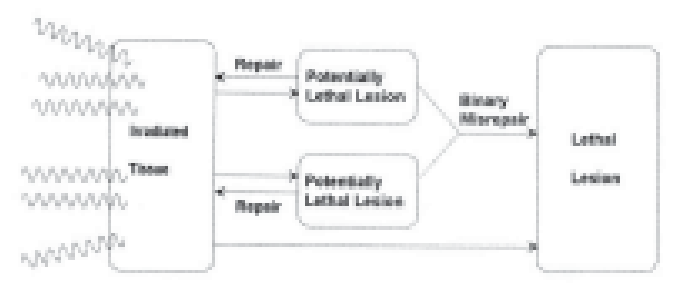

Fig.10 Lethal-Potentially-Lethal model.

Fig. 10 A biological model, consistent with a linear quadratic relationship between radiation dose and cell death, is necessary if the said relationship can give any value. One such model is the Lethal-Potential Lethal model. This concentrates on the lesion production and the interaction of lesions. In themselves insufficient to be lethal. The immediately lethal part of the model could relate to the linear part of the curve while the interaction of potentially lethal lesions could relate to the quadratic part of the curve.

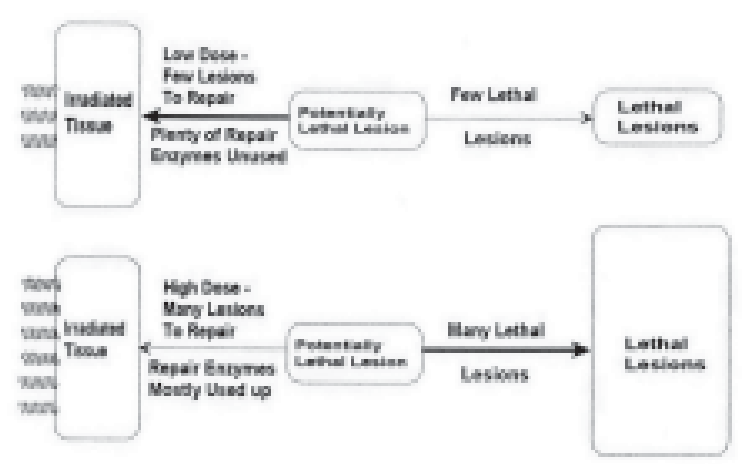

Fig. 11. Repair Saturation Model of Cell Killing. 
It is also consistant with a linear quadratic relationship between radiation dose and cell death. This concentrates entirely on the accumulating effect of increasing radiation dose on the enzymes responsible for repairing the damage done by radiation. In the upper part of this diagram the dose is low while in the lower part it is higher, indicating a gradual failure of repair capacity. Thus direct hits producing immediate lesions could account for the linear part of curve. The quadratic part of the curve could reflect the increasing failure of overloaded repair enzymes with increasing radiation dose.

\section{References:}

1. J. C. Ganz, Gamma knife Radiosurgery (Springer, Australia, 1997 )

2. Steve Webb, "The Physics of Three - Dimensional Radiation Therapy, Conformal Radiotherapy, Radiosurgery and Treatment Planning”,(Institute of Physics Publishing, Bristol and Philadelphia 1993)

3. Bazaraa and Shetty, "Nonlinear Programming -Theory and Algorithms”(John Wiley and Sons.Inc.Toranto,1993)

4. Spencer, Parker, Berry, England, Faulkner, Green, Holden, Middleton and Rogers, “Engineering Mathematics” Volume 2(Van Nostrand Reinhold Company Ltd., England, 1977)

5. A.Wu et al., " Physics of gamma knife approach on convergent beams in stereotactic radiosregery," International Journal of Radiation, Oncology, Biology, Physics 18, $941-949$ (1990).

6. J. C. Flickinger et al., "Treatment planning for gamma knife radiosurgery with multiple isocentres,” International Journal of Radiation, Oncology, Biology, Physics 18, 14951501(1990).

7. A. H. Maitz et al., "Quality assurance for gamma knife stereotactic radiosurgery,” International Journal of Radiation, Oncology, Biology, Physics 32, 1465-1471 (1995).

8. Q.J. Wu and J.D. Bourland, "Morphology-guided radiosurgery treatnent planning and optimization for multiple isocentres," Medical Physics 26,2151-2160 (1999).

9. G. Stucklschweiger, "Stereotaxic convergent irradiation with the gamma knife. A study of the possibilities for optimizing the dosage distribution," Strahlenther Onkol. 171,499-509 (1995).
10. H. Z. Shu et al., "Treatment planning optimization by quasiNewton and simulated annealing methods for gamma unit treatment system,” Phy.Med.Biol. 43,2795-2805(1998).

11. Y.Yan et al., "Clinical treatment planning optimization by Powell's method for gamma unit treatment system," International Journal of Radiation, Oncology, Biology, Physics 39, 247-254 (1997).

12. H. Shu et al., "Three-dimensional optimization of treatment planning for gamma unit treatment system.” Med. Phys. 25,2352-2357(1998).

13. L. Luo et al., "Optimizing computerized treatment planning for the gamma knife by source culling,” International Journal of Radiation, Oncology, Biology, Physics 45,13391346(1999).

14. R. A. Stone, V.Smith, and L. Verhey, "Inverse planning for the Gamma knife,” Med. Phys.20, 865(1993)

15. M. C. Ferris and D.M. Shepard, "Optimization of Gamma knife radiosurgery," presented at the proceedings of the DIMACS Workshop on Discrete Mathematical Problems and Medical Applications, Rutgers University, Piscataway, NJ, 1999.

16. H. M. Kooy, L.A. Nedzi, J. S. Loeftter , C. Cheng, E. Mannarino, and R. Siddon, "Treatment planning for stereotactic radiosurgery of intra-cranial lesions”. International Journal of Radiation Oncology, Biology and Physics, 21:683693,1991.

17. E. K.Lee, T. Fox, and I. Crocker. "Optimizing of radiosurgery treatment planning via mixed integer programming”. Medical physics 2000.

18. D.M.Shepard,M.C.Ferris, R. Ove, and L. Ma. "Inverse planning for gamma knife radiosurgery. Medical Physics,27:12,2000.

19. D.M.Shepard,M.C.Ferris, G. Olivera, and T. R. Mackie. Optimizing the delivery of radiation to cancer patients. SIAM REVIEW, 41: 721-744,1999.

20. S. Webb. Optimisation of conformal radiotherapy dose distributions by simulated annealing. Physics in Medicine and Biology, 34(10): 1349-1370,1989.

21. Faiz M. Khan. "The Physics of Radiation Therapy." LIPPINCOTT WILLIAMS AND WILKINS, Philadelphia, USA. $3^{\text {RD }}$ Edition.

22. "David M. Shepard, Lawrence S. Chin, Steven J. DiBiase, Shahid A. Naqvi, Jinho Lim, Michhael C. Ferris. Clinical implementation of an automated planning system for gamma knife radiosurgery”. Medical Physics,2001 